\title{
Transcriptome profiling of drought responsive noncoding RNAs and their target genes in rice
}

\author{
Pil Joong Chung ${ }^{1}$, Harin Jung ${ }^{1}$, Dong-Hoon Jeong ${ }^{3}$, Sun-Hwa Ha ${ }^{4}$, Yang Do Choi ${ }^{1,2}$ and Ju-Kon Kim*
}

\begin{abstract}
Background: Plant transcriptome profiling has provided a tool for understanding the mechanisms by which plants respond to stress conditions. Analysis of genome-wide transcriptome will provides a useful dataset of drought responsive noncoding RNAs and their candidate target genes that may be involved in drought stress responses.

Results: Here RNA-seq analyses of leaves from drought stressed rice plants was performed, producing differential expression profiles of noncoding RNAs. We found that the transcript levels of 66 miRNAs changed significantly in response to drought conditions and that they were negatively correlated with putative target genes during the treatments. The negative correlations were further validated by qRT-PCR using total RNAs from both droughttreated leaves and various tissues at different developmental stages. The drought responsive miRNA/target pairs were confirmed by the presence of decay intermediates generated by miRNA-guided cleavages in Parallel Analysis of RNA Ends (PARE) libraries. We observed that the precursor miR171f produced two different mature miRNAs, miR171f-5p and miR171f-3p with 4 candidate target genes, the former of which was responsive to drought conditions. We found that the expression levels of the miR171f precursor negatively correlated with those of one candidate target gene, but not with the others, suggesting that miR171f-5p was drought-responsive, with Os03g0828701-00 being a likely target. Pre-miRNA expression profiling indicated that miR171f is involved in the progression of rice root development and growth, as well as the response to drought stress. Ninety-eight IncRNAs were also identified, together with their corresponding antisense transcripts, some of which were responsive to drought conditions.
\end{abstract}

Conclusions: We identified rice noncoding RNAs (66 miRNAs and 98 IncRNAs), whose expression was highly regulated by drought stress conditions, and whose transcript levels negatively correlated with putative target genes.

Keywords: Oryza sativa, Drought stress, IncRNAs, miRNA, Non-coding RNA, Putative target genes, RNA-seq

\section{Background}

There is growing concern regarding current and future environmental changes worldwide, such as increases in average air and sea temperatures and altered rainfall patterns, and the abiotic stresses that they impose on biological systems $[1,2]$. Plant adaptations to such stresses involve complex signal transduction pathways [3], and elucidating the associated gene expression networks [4], in order to develop strategies to enhance the stress

\footnotetext{
* Correspondence: jukon@snu.ac.kr

${ }^{1}$ Graduate School of International Agricultural Technology and Crop Biotechnology Institute/GreenBio Science \& Technology, Seoul National University, Pyeongchang 25354, Korea

Full list of author information is available at the end of the article
}

tolerance of crops [5-7], is an important objective of agricultural biotechnology.

Many studies have investigated plant stress tolerance using transcriptional profiling, thereby revealing differences between control and stress-treated plants in the relative expression levels of genes encoding stress response regulators and their target proteins [4, 8]. However, while typically more than $90 \%$ of a eukaryotic genome is transcribed, only $1-2 \%$ is translated into proteins [9], and indeed, in addition to stress-inducible regulatory proteins and transcription factors, microRNAs (miRNAs) are also known to regulate plant stress responses [10-12]. miRNAs are a class of small noncoding RNAs that regulate gene expression at the posttranscriptional level by mRNA cleavage or translational 
inhibition of the target gene [13]. There are currently $>10,000$ plant miRNA sequences from $>120$ plant species in the miRBase database (www.mirbase.org) [14] including 713 from rice (Oryza sativa) miRNA sequences. Several miRNAs have been reported to regulate drought-responsive genes $[10,15,16]$, and it has been shown that rice miR159, miR169, miR395 and miR474 are drought-inducible, while the expression of miR156, miR168, miR170, miR172, miR396, miR397 and miR408 is suppressed by drought $[13,16]$. In addition, miR171 and miR319 expression is either increased or repressed, depending on the specific drought conditions [15]. Drought-induced miRNAs downregulate their target transcripts, whereas drought-induced suppression of miRNAs results in the increased accumulation of their target transcripts $[17,18]$. For example, miR169 is downregulated under drought stress in Arabidopsis thaliana, whereas its target gene, NFYA5, is drought-induced [19]. Phenotypic analysis of mutants, or transgenic plants in which the expression of either stress-responsive miRNAs or their target genes have been manipulated, has been used to determine the role of miRNAs under different stress conditions $[20,21]$.

Another class of noncoding RNAs are the long noncoding RNAs (lncRNAs), which can be classified into five categories: i) sense and ii) antisense, when there is overlap of different transcripts in the same, or opposite, strand, respectively; iii) bidirectional, when the expression of an lncRNAs and a neighboring coding transcript on the opposite strand is initiated in close genomic proximity; iv) intronic, when it is derived wholly from within an intron of a second transcript; and v) intergenic, when it lies within the genomic interval between two genes [22]. Numerous lncRNAs have been associated with responses to abiotic stress, such as the expression of 1,832 lncRNAs that were reported to be regulated by various abiotic stresses in A. thaliana [23], 125 lncRNAs that were identified under drought and heat stress conditions in wheat (Triticum aestivum) [24] and several drought-responsive and tissue-specific maize (Zea mays) lncRNAs [25].

In this current study, RNA-sequencing (RNA-seq) transcript profiling was used to evaluate the levels of noncoding RNAs, including pri-miRNAs and lncRNAs, in well-watered control and drought-treated rice plants. A total of 66 drought-responsive miRNA precursors (24 drought-inducible and 42 drought-repressible), which have not previously been characterized in rice, were identified. The expression levels of some of these were shown, by qRT-PCR, to have a negative correlation with the expression of their candidate target genes. In addition, Parallel Analysis of RNA Ends (PARE) libraries from various rice tissues enabled the identification of decay intermediates generated by miRNA-guided cleavages [26], and a total of 98 drought-responsive lncRNAs and their sense or antisense transcripts were detected. The combined data sets suggest potential roles for specific rice miRNAs under drought conditions.

\section{Results \\ Exposure of rice plants to conditions that mimic natural drought stress}

Rice plants were grown in a greenhouse for 5 weeks before being subjected to drought stress. To mimic natural drought stress, drought conditions were imposed by withholding water for $3 \mathrm{~d}$, until a soil water content of < $10 \%$ was measured. Leaves of drought stressed plants were compared to those of control plants grown under normal irrigation conditions. After a day, the soil moisture content dropped to $50 \%$ of the initial soil capacity and rice plants started to show visual symptoms of drought-induced damage, such as leaf rolling (Fig. 1a and Fig. 1b). All the leaves from drought-treated plants showed a greater degree of leaf rolling as the level of drought stress increased. Consequently, the rice plants were severely affected by drought after $3 \mathrm{~d}$ (Fig. 1a). In addition to the phenotypic assessment, we measured the expression of the Dip1 (Dehydration stress-inducible protein 1; Os02g0669100) and RbcS1 (Small subunit of rubisco; Os12g0274700) genes, whose expression has been reported to be drought-inducible and droughtrepressed, respectively [27]. Dip1 expression was observed to increase at $1 \mathrm{~d}$, and continued to increase up to $3 \mathrm{~d}$, whereas transcript levels of $R b c S 1$ progressively decreased until $3 \mathrm{~d}$ after the imposition of drought conditions (Fig. 1c).

\section{RNA-seq analysis}

Total RNA was extracted from the leaves of drought treated and well-watered plants and used to construct four RNA-seq libraries: one library from a well-watered control $(\mathrm{C})$ and three libraries from drought-treated leaves ( 1 to $3 \mathrm{~d}$ ). These were sequenced using an Illumina Hi-seq 2500 to identify differences in expression profiles among the different libraries. Sequence read information is summarized in Additional file 1: Table S1. Approximately 492 million single-end sequence reads were obtained and after quality trimming a total of 254 million sequence reads remained, corresponding to $24,667,603,889$ bp (49 \%). A flow chart of the sequencing process is shown in Additional file 2: Figure S1. A total of $81 \%$ of the reads could be mapped to predicted gene regions. Raw sequence reads were trimmed to remove adaptor sequences and those with a quality lower than Q20 were also removed using the clc mapping tool (clc_ref_assemble 6 in the CLC ASSEMBLY CELL package). 


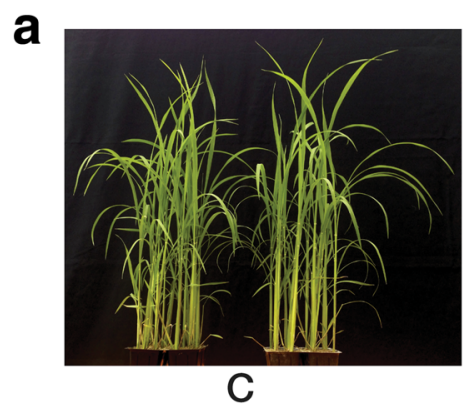

b



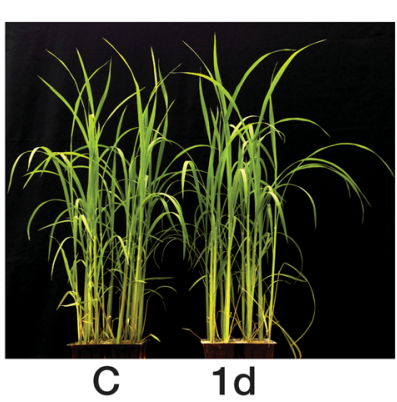
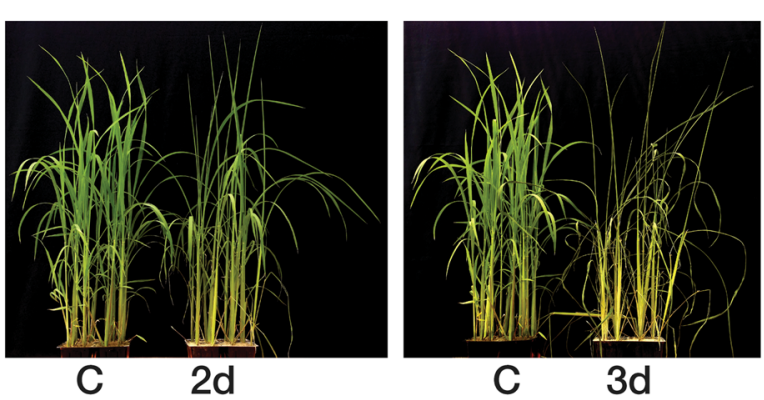

C
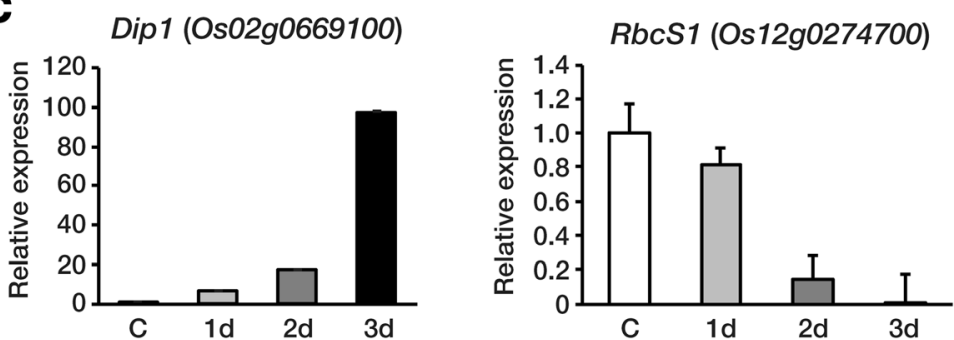

Fig. 1 Drought response phenotype of rice in the vegetative state. a The phenotypic effect of progressive drought on wild type rice (Oryza sativa cv. IImi) at the vegetative growth stage. Drought stress was initiated 40 days after germination, and the plants shown are a well-watered control and at day 1, 2 and 3 after drought initiation. $\mathbf{b}$ Decrease in soil water content during drought treatment. Soil moisture in the pots was monitored using a SM150 Soil Moisture Sensor (Delta-T Devices Ltd). Volts (mV) is the SM150 output value. Blue bar, control; Red bar, drought condition. The

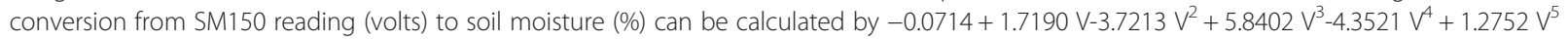
(Delta-T Devices Ltd). c The transcript levels of Dip 1 and RbcS1 in the leaves of drought-treated and well-watered control plants over a time course of exposure to drought were measured by qRT-PCR analysis. Values shown are the means \pm SD of three independent experiments and are presented relative to the results from the control. Dip1 (Dehydration Stress-Inducible Protein1, Os02g0669100) and RbcS1 (Small subunit of Rubisco, Os12g0274700) served as stress marker genes

Drought responsive genes were defined those that were differentially expressed between well-watered and drought-treated leaves, and we observed that among these genes, approximately twice as many were downregulated by drought as were up-regulated in the $2 \mathrm{~d}$ and $3 \mathrm{~d}$ samples (Additional file 3: Figure S2). Far fewer genes were up-regulated in the $1 \mathrm{~d}$ sample. Of the 44,553 genes that could be annotated using the RAP-DB database (http://rapdb.dna.affrc.go.jp), 1,963 and 2,286 were up- and down-regulated, respectively, by more than 2-fold upon drought treatment for $1 \mathrm{~d}$. Similarly, 8,070 and 12,518 genes were up- and down-regulated, respectively, after $2 \mathrm{~d}$, and 7,888 and 17,746 genes, respectively, after $3 \mathrm{~d}$. Of the genes identified as differentially expressed between treatments, 853 and 479 were up- and downregulated, respectively, in all three drought treated samples (Additional file 4: Tabular data 1).

The assembled contigs were annotated using the gene ontology $(\mathrm{GO})$ database BLAST mapping function (BLAST2GO) at the EMBL-EBI website (http:// www.ebi.ac.uk/QuickGO/GAnnotation). Additional file 5: Figure $\mathrm{S} 3$ shows the genes that could be assigned at least one GO term in the three main GO categories, 'biological process', 'cellular component' and 'molecular function'.

\section{Drought-responsive miRNAs and their candidate target genes}

To date, 592 rice miRNA precursors (pre-miRNAs) encoding 713 mature miRNAs have been reported (www.mirbase.org), and in this current study we identified a total of 113 pre-miRNAs in the RNA-seq data sets. Of those, 26 pre-miRNAs were constitutively expressed under both normal and drought conditions at high levels, whereas 21 pre-miRNAs were expressed at low levels (Additional file 6: Table S3). In addition, the expression levels of 24 pre-miRNAs increased considerably upon exposure to drought stress conditions, while those of 42 were substantially decreased (Additional file 7: Table S2). These drought-responsive miRNAs and their putative target genes, predicted by the web tool psRNATarget (http://plantgrn.noble.org/psRNATarget/), are listed in Additional file 8: Tabular data 2. For 18 of the pre-miRNAs that were highly up-regulated in response to drought stress, their putative target genes showed a concomitant decrease in transcript levels. Conversely, for 20 precursor miRNAs that were strongly down-regulated by the drought treatment, a concomitant increase in transcript levels of their putative target genes was observed (in Additional file 8: Tabular data 2). To validate the RNA-seq results and the inverse 
correlations in expression levels between the miRNAs and their target genes, qRT-PCR was carried out using total RNAs from control and drought-treated leaves. Expression levels of the miRNAs and their candidate target genes were again seen to be inversely correlated (Fig. 2), consistent with their expected function in cleaving the target mRNAs. qRT-PCR was also used to determine the correlation in expression of the precursor and mature miRNAs, and we observed that the expression patterns of the drought-responsive miR171f-5p, miR399k, miR818b and miR156d precursors correlated well with those of the mature miRNAs (Fig. 2a). Interestingly, while pre-miR171f and miR171f-5p showed a drought-inducible expression pattern, the expression of miR171f-3p, which is another mature miRNA derived from pre-miR171f, was affected by drought. However, this could be due to the fact that the miR171f-3p sequence is also encoded by other members of the miR171 family, such as pre-miR171b, pre-
miR171c, pre-miR171e and pre-miR171f, which are also not responsive to drought. It is also possible that processing of pre-miR171f to generate miR171-5p or miR171-3p is differentially regulated by drought. Since miR-171f-5p and miR-171f-3p have different sets of target genes, we measured the expression levels of the miR171f precursor and the putative target transcripts (Os03g0828701-00 and Os12g0571900-01 for miR-171f-5p; Os09g0555600-01 and Os05g0417100-01 for miR-171f-3p) in various rice tissues at different developmental stages by qRT-PCR (Fig. 3) [28]. The precursor miR-171f accumulated at high levels in roots, coleoptiles and flowers. Conversely, transcripts of Os03g0828701-00, a target of miR-171f-5p, were observed in leaves, but not in roots and flowers. Thus, the expression pattern of the miR-171f precursor has an inverse correlation with that of its corresponding target gene, Os03g0828701-00, but not with the other predicted target genes, Os12g0571900-01, Os09g0555600-01 and

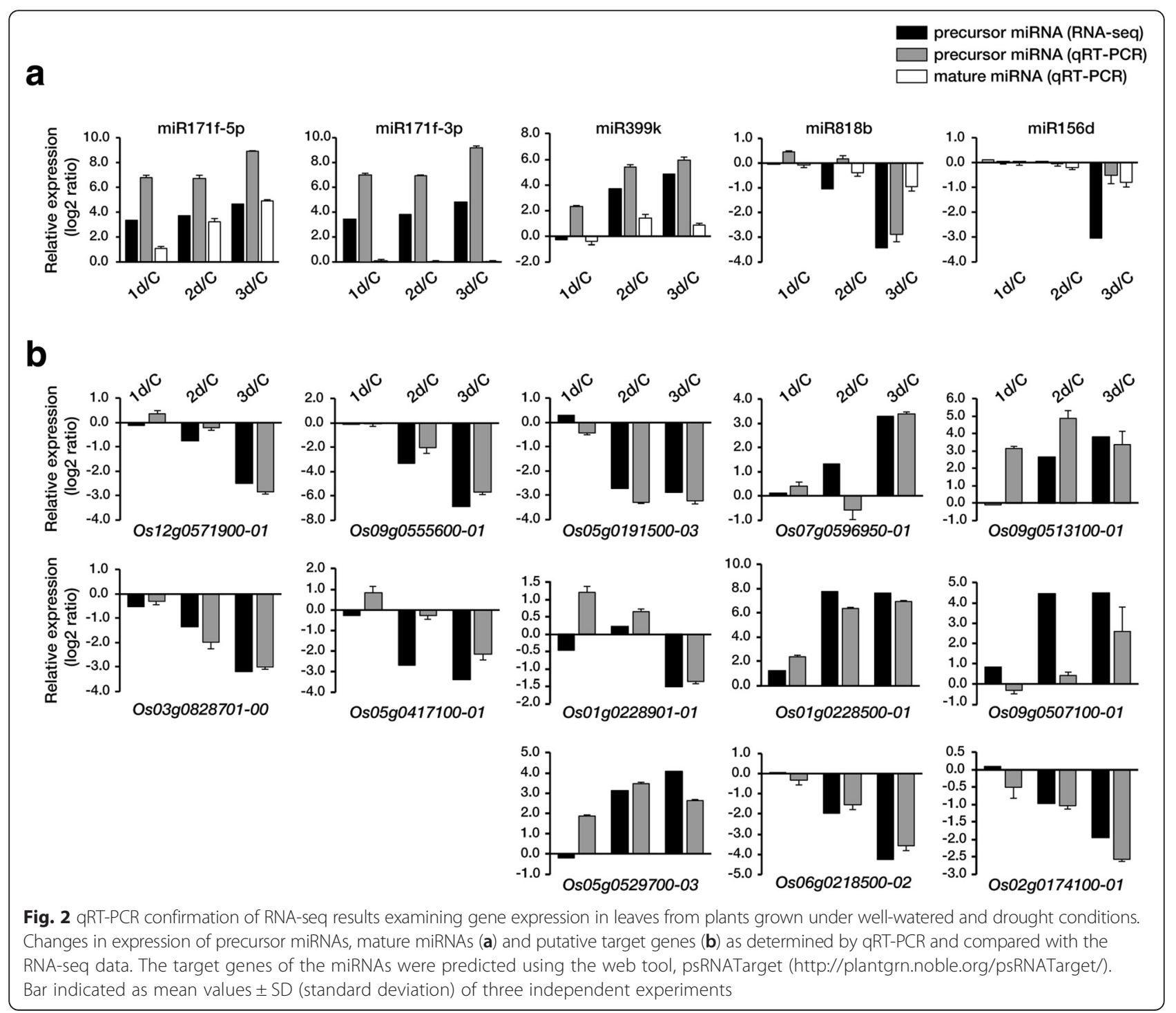





Fig. 3 Expression analysis of the drought-responsive miRNA precursor miR-171f and its putative target genes (Os03g0828701-00, Os12g0571900-01, Os09g0555600-01 and Os05g0417100-01) in various plant tissues at different developmental stages. Rice seeds were germinated and grown on MS (Murashige and Skoog) medium in the dark for $3 \mathrm{~d}$ ( $3 \mathrm{DAG}$, day after germination) and then in the light for $1 \mathrm{~d}$ at $28^{\circ} \mathrm{C}(4 \mathrm{DAG})$. Seedlings were then transplanted into soil pots, and grown in the greenhouse for $10 \mathrm{~d}, 15 \mathrm{~d}, 1$ month and 2 month until meiosis (meiosis), just prior to heading (before heading, $\mathrm{BH}$ ) and right after heading (after heading, AH). qRT-PCR analyses of each gene were performed with the indicated tissues at the different developmental stages. Rice Ubi1 (AK121590) was used as an internal control. C, coleoptiles; R, roots; L, leaves; FL, flag leaves; F, flowers (panicles). Bar indicated as mean values \pm SD (standard deviation) of three independent experiments

Os05g0417100-01. Considering the results presented in Figs. 2 and 3 , we concluded that miR-171f-5p is drought-responsive, with Os03g0828701-00 being a likely target gene.

Parallel Analysis of RNA Ends (PARE), also known as RNA degradome analysis, enables high-throughput miRNA target identification. To validate predicted targets of the drought-responsive miRNAs, rice PARE data were obtained from the NCBI Gene Expression Omnibus with accession numbers GSM455938, GSM455939, GSM476257 and GSM434596 (http://www.ncbi.nlm.nih.gov/geo/) [29]. PARE sequences matching to cleavage products, starting between base 10 and 11 from the $5^{\prime}$ end of the predicted miRNA pairing, were considered to be evidence of miRNA-guided cleavage (Table 1). In total, 32 target cleavages guided by 21 drought-responsive miRNAs were identified.

\section{Drought-responsive IncRNAs and their Natural Antisense Transcripts (NATs)}

In this study, 98 drought-responsive lncRNAs (31 upand 67 down-regulated, respectively, with a $\log _{2}$ ratio $\geq$ 2.0 and $\leq-2.0$ ) with over $1 \mathrm{~kb}$ in length and their cognate antisense transcripts were identified (Additional file 9: Table S4 and Additional file 10: Tabular data 3). A subset of the IncRNAs comprise the class 'Natural 
Table 1 Drought responsive precursor miRNAs with their candidate target genes and their expression patterns

\begin{tabular}{|c|c|c|c|c|c|c|c|c|c|c|c|c|c|}
\hline \multirow[t]{2}{*}{ Gene ID } & \multicolumn{4}{|l|}{${ }^{\mathrm{a}} \mathrm{RPKM}$} & \multicolumn{3}{|c|}{${ }^{\mathrm{b}}$ Log2 Ratio } & \multirow[t]{2}{*}{${ }^{\circ}$ Mature miRNA/target sequence } & \multirow[t]{2}{*}{${ }^{d}$ PARE sequence } & \multicolumn{4}{|c|}{ PARE libraries } \\
\hline & $\bar{C}$ & d1 & $\mathrm{d} 2$ & d3 & $1 \mathrm{~d} / \mathrm{C}$ & $2 \mathrm{~d} / \mathrm{C}$ & $3 \mathrm{~d} / \mathrm{C}$ & & & $\overline{{ }^{e} \mathrm{~A}}$ & $f_{B}$ & ${ }^{9} \mathrm{C}$ & $\frac{h_{D}}{h_{1}}$ \\
\hline \multicolumn{14}{|l|}{ Inducible miRNAs/targets } \\
\hline miR-399k & 119,291 & 99,410 & $1,530,906$ & $3,519,097$ & -0.26 & 3.68 & 4.88 & 3'-GCCCCGUUUAAAGGAAACCGU-5' & & & & & \\
\hline Os05g0557700-01 & 25,889 & 23,611 & 8,802 & 9,104 & -0.13 & -1.56 & -1.51 & 737-CUGGGCAAAUCUCCUUUGGCA-757 & TCCTITGGCAAAATACCTAT & 0 & 0 & 1 & 1 \\
\hline miR-415 & 234,165 & 109,765 & 160,988 & $1,529,388$ & -1.09 & -0.54 & 2.71 & $3^{\prime}-$ GACGAGACGAAGACAAGACAA-5' & & & & & \\
\hline Os04g0550200-01 & 9,337 & 2,36 & 1,749 & 273 & -1.98 & -2.42 & -5.10 & 789-UUACUCUGCUUCUGCUCUGUU-809 & CTGCTCTGTTCTCTITCTTC & 0 & 0 & 0 & 1 \\
\hline Os10g0500500-01 & 13,806 & 11,519 & 7,428 & 3,613 & -0.26 & -0.89 & -1.93 & 976-UGCUCUGCAUUUCUUCUGUU-995 & TTCTTCTGTTACTCATTCGA & 0 & 2 & 0 & 0 \\
\hline miR-168a & 329,822 & 678,870 & $1,513,995$ & 981,069 & 0.11 & 1.27 & 0.64 & 3'-AGGGCUAGACGUGGUUCGCU-5' & & & & & \\
\hline Os02g0831600-01 & 5,715 & 5,283 & 1,584 & 687 & -0.11 & -1.85 & -3.06 & 446-UCCCGAGCUGCGCCAAGCAA-465 & CGCCAAGCAATAATGGAAGC & 0 & 0 & 2 & 7 \\
\hline Os03g0687000-02 & 11,281 & 10,412 & 3,293 & 1,896 & -0.12 & -1.78 & -2.57 & 1807-UCAUGAUCUGCGCCAAGUGG-1826 & CGCCAAGTGGTACAGGTTCA & 0 & 0 & 0 & 1 \\
\hline Os03g0687000-01 & 9,006 & 8,713 & 2,571 & 1,591 & -0.05 & -1.81 & -2.50 & 1905-UCAUGAUCUGCGCCAAGUGG-1924 & CGCCAAGTGGTACAGGTTCA & 0 & 0 & 0 & 1 \\
\hline miR-821c & 27,730 & 47,537 & 376,336 & 364,452 & 0.78 & 3.76 & 3.72 & 3'-AGUUGAAAAAACAACUACUGAA-5' & & & & & \\
\hline Os10g0412600-01 & 14,944 & 16,110 & 4,884 & 334 & 0.11 & -1.61 & -5.48 & 685-UGAACUUUUUUAUUGGUGAUUC-706 & TTGGTGATTCCCTCTAATGT & 0 & 0 & 0 & 1 \\
\hline miR-171f-3p & 8,297 & 82,972 & 107,863 & 207,429 & 3.32 & 3.70 & 4.64 & 3'-CUAUAACCGUGCCGAGUUAGU-5' & & & & & \\
\hline Os09g0555600-01 & 2,031 & 1,926 & 380 & 60 & -0.08 & -2.42 & -5.07 & 1488-GGUAUUGGCAUUGCUCAAUUA-1508 & TGCTCAATTATGGGCTAAAG & 0 & 0 & 0 & 1 \\
\hline miR-816 & 150,534 & 95,795 & 301,069 & 136,849 & -0.65 & 1.00 & -0.14 & 3'-CAACAUCAUUUUAUACAGUG-5' & & & & & \\
\hline Os01g0338100-00 & 1,722 & 1,227 & 701 & 534 & -0.49 & -1.30 & -1.69 & 277-AUUGUUGUAGAAUAUGUCAC-296 & AATATGTCACTGACCTGGTC & 0 & 0 & 0 & 1 \\
\hline miR-166c-5p & 16,860 & 50,580 & 25,290 & 42,150 & 1.58 & 0.58 & 1.32 & 3'-GAGCCUGGUCUGUUGUAAGG-5' & & & & & \\
\hline Os03g0823100-01 & 41,418 & 36,220 & 21,357 & 6,526 & -0.19 & -0.96 & -2.67 & 784-CUUGGACCAGCCAAUAUUUU-803 & CCAATATTTTCCTTCTATT & 0 & 0 & 1 & 1 \\
\hline miR-166c-3p & 16,860 & 50,580 & 25,290 & 42,150 & 1.58 & 0.58 & 1.32 & $3^{\prime}-C C C U U A C U U C G G A C C A G G C U-5^{\prime}$ & & & & & \\
\hline Os12g0612700-01 & 667 & 751 & 573 & 121 & 0.17 & -0.22 & -2.47 & 874-UGGGAUGAAGCCUGGUCCGG-893 & CCTGGTCCGGATTCCATTGG & 0 & 0 & 53 & 73 \\
\hline miR-167g & - & 25,701 & 89,953 & 12,850 & - & - & - & 3'-GUCUAGUACGACCGUCGAAGU-5' & & & & & \\
\hline miR-167b & 6,465 & & 19,394 & 12,929 & - & 1.58 & 1.00 & 3'-UCUAGUACGACCGUCGAAGU-5' & & & & & \\
\hline Os06g0129100-01 & 21,789 & 14,688 & 2,477 & 275 & -0.57 & -3.14 & -6.31 & 1085-UGUUCAUGCCGGCAGCUUCA-1104 & GGCAGCTTCAGGCTCCAGGT & 0 & 0 & 0 & 10 \\
\hline Os07g0481400-01 & 10,354 & 8,139 & 4,789 & 1,387 & -0.35 & -1.11 & -2.90 & 2770-UAGAUCAUGCUGACAGCCUCA-2790 & GACAGCCTCAAAACAATTGA & 0 & 0 & 1 & 6 \\
\hline miR-159b & 11,210 & 67,260 & 67,260 & - & 2.58 & 2.58 & - & 3'-GUCUCGAGGGAAGUUAGGUUU-5' & & & & & \\
\hline Os06g0605600-01 & 3,381 & 3,623 & 4,557 & 934 & 0.10 & 0.43 & -1.86 & 403-UAGAGCUCCCUUCACUCCAAU-423 & TCACTCCAATATCCCAACTA & 0 & 3 & 0 & 16 \\
\hline Os03g0683866-00 & 5,268 & 5,001 & 4,429 & 1,662 & -0.07 & -0.25 & -1.66 & 1320-UAAAGCUGCCUUCAGUCCAGA-1340 & TCAGTCCAGAATATGGGCTT & 0 & 0 & 0 & 1 \\
\hline miR-159f & 5,605 & 11,210 & 28,025 & - & 1.00 & 2.32 & - & 3'-AUCUCGAGGGAAGUUAGGUUC-5' & & & & & \\
\hline Os06g0605600-01 & 3,381 & 3,623 & 4,557 & 934 & 0.10 & 0.43 & -1.86 & 403-UAGAGCUCCCUUCACUCCAAU-423 & TCACTCCAATATCCCAACTA & 0 & 3 & 0 & 16 \\
\hline miR-169f & - & 6,198 & 43,389 & 198,351 & -- & - & - & 3'-AUCCGUUCAGUAGGAACCGAU-5' & & & & & \\
\hline Os02g0776400-01 & 1,807 & 1,507 & 469, & 60 & -0.26 & -1.94 & -4.91 & 983-UAGGCAAUUCAUCCUUGGCUU-1003 & TCCTTGGCTTAAGTTTCATG & 6 & 5 & 6 & 66 \\
\hline Os03g0411100-01 & 12,413 & 14,677 & 4,257 & 2,061 & 0.24 & -1.54 & -2.59 & 1242-UGGCAAUUCAUCCUUGGCUU-1261 & TCCTTGGCTTATGAAGTATC & 28 & 42 & 37 & 156 \\
\hline
\end{tabular}


Table 1 Drought responsive precursor miRNAs with their candidate target genes and their expression patterns (Continued)

\begin{tabular}{|c|c|c|c|c|c|c|c|c|c|c|c|c|c|}
\hline miR-156i & - & - & 23,416 & 23,416 & -- & -- & -- & 3'-CACGAGUGAGAGAAGACAGU-5' & & & & & \\
\hline Os06g0663500-00 & 3,829 & 3,070 & 3,323 & 867 & -0.32 & -0.20 & -2.14 & 749-GUGCUCUCUCUCUUCUGUCA-768 & TCTTCTGTCAGCTAGTTCAA & 0 & 5 & 1 & 35 \\
\hline Os02g0174100-01 & 2,114 & 2,279 & 1,071 & 564 & 0.11 & -0.98 & -1.95 & 2221-GUGCUCUCUCUCUUCUGUCA-2240 & TCTTCTGTCATCTAGTTCTT & 0 & 2 & 0 & 11 \\
\hline Os02g0139400-01 & 13,403 & 16,632 & 13,100 & 1,392 & 0.31 & -0.03 & -3.27 & 1869-AUGCUCUCUCUCUUCUGUCA-1888 & TCTTCTGTCAATCGATTCAG & 0 & 5 & 25 & 34 \\
\hline \multicolumn{14}{|c|}{ Repressible miRNAs / targets } \\
\hline miR-530 & $1,433,087$ & 241,496 & 231,823 & 84,299 & -1.77 & -2.63 & -4.09 & 3'-AUCCACGUCCACGUUUACGU-5' & & & & & \\
\hline Os04g0603200-01 & 28,344 & 24,091 & 55,324 & 69,156 & -0.23 & 0.96 & 1.29 & 653-CAGAUGAAGGUGCAAAUGCA-672 & TGCAAATGCAGGAGCTGTAA & 0 & 0 & 0 & 1 \\
\hline Os03g0296700-02 & 334 & 434 & 534 & 568 & 0.38 & 0.68 & 0.77 & 440-UGGAUGCUGGUGCAGAUGCA-459 & TGCAGATGCACCGTTCTGAT & 0 & 0 & 1 & 0 \\
\hline miR-399e & 169,670 & 107,160 & 17,860 & 8,930 & -0.66 & -3.25 & -4.25 & 3'-CCCGUUUAGAGG-AAACCGU-5' & & & & & \\
\hline Os04g0415000-01 & 5,599 & 8,625 & 25,400 & 18,132 & 0.62 & 2.18 & 1.70 & 491-GGGCAAUUCUCCGUUUGGCA-510 & CCGTTTGGCAGAAGATCAAC & 0 & 0 & 0 & 1 \\
\hline miR-156f & $1,501,297$ & $1,370,996$ & 657,172 & 436,226 & -0.13 & -1.19 & -1.78 & 3'-CACGAGUGAGAGAAGACAGU-5' & & & & & \\
\hline miR-156d & $1,070,078$ & $1,159,932$ & $1,110,921$ & 130,697 & 0.12 & 0.05 & -3.03 & 3'-CACGAGUGAGAGAAGACAGU-5' & & & & & \\
\hline miR-156g & 136,247 & 364,020 & 657,790 & 19,159 & 0.09 & -0.45 & -1.91 & 3'-CACGAGUGAGAGAAGACAGU-5' & & & & & \\
\hline miR-156j & 351,247 & 364,020 & 657,790 & 19,159 & 0.05 & 0.91 & -4.20 & 3'-CACGAGUGAGAGAAGACAGU-5' & & & & & \\
\hline Os01g0922600-01 & 1,610 & 2,024 & 20,929 & 4,295 & 0.33 & 3.70 & 1.42 & 620-GUGCUCUCUCUCUUCUGUCA-639 & TCTTCTGTCAGACAACCCCA & 0 & 0 & 2 & 19 \\
\hline Os09g0507100-00 & 42 & 75 & 941 & 950 & 0.83 & 4.47 & 4.49 & 1034-GUGCUCUCUCUCUUCUGUCA-1053 & TCTTCTGTCATCCCCGGCCA & 0 & 0 & 2 & 1 \\
\hline miR-815a & 25,391 & - & 12,696 & 12,696 & - & -1.00 & -1.00 & 3'-GGUUAGAGGAGUUAGGGGAA-5' & & & & & \\
\hline miR-815c & 511,525 & 531,986 & 225,071 & 71,613 & 0.06 & -1.18 & -2.84 & 3'-GGUUAGAGGAGUUAGGGGAA-5' & & & & & \\
\hline Os08g0465800-01 & 9,970 & 9,526 & 61,668 & 74,744 & -0.07 & 2.63 & 2.91 & 1896-CCAAUCUCCUUCCUCCUCUU-1915 & ТССТССТСТIITTAATCTCT & 0 & 0 & 0 & 1 \\
\hline miR-159a & 395,153 & 360,286 & 96,851 & 65,859 & -0.13 & -2.03 & -2.58 & 3'-UCUCGAGGGAAGUUAGGUUU-5' & & & & & \\
\hline Os03g0331700-02 & 7,076 & 3,394 & 22,641 & 50,780 & -1.06 & 1.68 & 2.84 & 1513-UGAGUUCCCUUCAUUCCAAA-1532 & TCATTCCAAAAGCTTAATTG & 0 & 0 & 0 & 1 \\
\hline miR-393b & 151,675 & 175,623 & 127,726 & 31,932 & 0.21 & -0.25 & -2.25 & 3'-UAGUUACGCUAGGGAAACCU-5' & & & & & \\
\hline Os05g0150500-00 & 5,537 & 4,773 & 20,300 & 36,796 & -0.21 & 1.87 & 2.27 & 1556-GACAAUGCGAUCCCUUUGGA-1575 & TCCCTTTGGATGTCGTCGTG & 16 & 18 & 88 & 655 \\
\hline miR-528 & 227,512 & 287,384 & 23,949 & - & 0.34 & -3.25 & - & 3'-GAGGAGACGUACGGGGAAGGU-5' & & & & & \\
\hline Os07g0570550-00 & 290 & 522 & 1,334 & 1,566 & 0.85 & 2.20 & 2.43 & 180-CUCCUCUGC-UGCCCCUUCCA-199 & GCCCCTTCCATGGCGCCCGC & 0 & 0 & 205 & 0 \\
\hline miR-166d & 92,729 & 33,720 & 84,299 & 16,860 & -1.46 & -0.14 & -2.46 & 3'-CCCUUACUUCGGACCAGGCU-5' & & & & & \\
\hline Os03g0640800-01 & 1,189 & 1,224 & 4,376 & 8,977 & 0.04 & 1.88 & 2.92 & 956-UGGGAUGAAGCCUGGUCCGG-975 & CCTGGTCCGGATTCCATTGG & 0 & 0 & 53 & 73 \\
\hline Os10g0480200-02 & 1,638 & 1,282 & 2,956 & 3,158 & -0.35 & 0.85 & 0.95 & 922-UGGGAUGAAGCCUGGUCCGG-941 & CCTGGTCCGGATTCGTTTGG & 0 & 3 & 12 & 179 \\
\hline
\end{tabular}

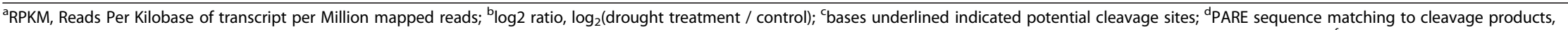
starting between base 10 and 11 from the 5 ' end of the predicted miRNA pairing; ${ }^{e} A$ (SC938), PARE library of rice wild type seedling degradome, GSM455938 (GEO Accession number); ${ }^{\prime} B$ (INF939), PARE library of rice wildtype inflorescence degradome, GSM455939; ${ }^{9} \mathrm{C}$ (INF9311a), PARE library of rice inflorescence (93-11) wildtype degradome, GSM476257; ${ }^{\mathrm{h} D}$ (NPBs), PARE library of rice 3-week-old seedlings wildtype degradome, GSM434596 [29] 
Antisense Transcripts' (NATs), which are complementary to other endogenous transcripts of coding or noncoding genes. These can be transcribed in cis from the same genomic locus as the target mRNA, or in trans from a separate locus. Of the 98 lncRNAs, 58 pairs were determined to be cis-NATs, i.e., two or more genes within the NAT pair that are located on the opposite strands of the same genomic locus. Additional 6 regions of bidirectional transcription were also found, which were arranged in a convergent orientation to the $5^{\prime}$ end or with the 3' end overlapping. Additionally, 22 intergenic and 5 sense lncRNAs (Additional file 9: Table S4) were identified, and we determined that the expressions of most of the lncRNAs was consistent with expression of the nearby coding or noncoding transcripts, while the expression of two NATs and their candidate target genes were inversely correlated: the NAT Os02g0250700-01 and its candidate target gene Os02g0250600-01 (late embryogenesis abundant protein, LEA), and the NAT Os 02g0180800-01 and its target gene Os02g0180700-01 (cinnamoyl-CoA reductase). The former pair shows a head-to-head genomic configuration while the latter shows a tail-to-tail configuration.

\section{Discussion}

Compared with natural drought conditions, where dehydration is typically gradual and progressive, experimental treatments to induce drought are often relatively severe and/or rapid. For example, widely used methods involve air drying with excised leaf disc or treating them with polyethylene glycol $[30,31]$. Such treatments are liable to cause osmotic stress rather than drought stress, and indeed it can be difficult to distinguish between these types of stress. In this current study, we sought to analyze the molecular response of rice plants subjected to a mild drought stress, thereby mimicking natural drought conditions. Transcriptome profiling was performed of leaves from rice plants grown at either $75 \%, 40 \%, 10 \%$ or $7 \%$ residual soil moisture content (Fig. 1), where drought stress damage was carefully monitored using the expression of Dip1 and $R b c S 1$ as markers for drought-inducible and drought-sensitive expression, respectively. We then examined the RNAseq data to identify differentially expressed genes involved in drought responses.

Amongst the genes that were found to be associated with the drought response and that were differentially expressed between well-watered and water-deficit conditions, we identified both drought induced genes, including late embryogenesis abundant (Os06g0324400-01, Os03g032290000, Os06g0110200-01), calcium-dependent membrane targeting domain protein (Os04g0476600-01), and drought repressed genes, such as A-type response regulator, (Os11 g0143300-01, Os12g0139400-01) (Additional file 4: Tabular data 1). In addition, a total of 66 drought-responsive premiRNAs were identified, 24 which were drought-induced and 42 of which were drought-repressed by more than 2 fold. Of the 66 rice pre-miRNAs, 41 are identified as being drought-responsive for the first time in this study (Table 1). Sixty-six pre-miRNAs could be assigned to 29 miRNA families, while 10 did not belong to any family. Interestingly, two members of the miR399 family, pre-miR399k and pre-miR399d, were up-regulated by drought stress, while other two members, pre-miR399e and pre-miR399i, were down-regulated by drought stress. Similarly, some members of the miR156, miR159, miR167 and miR169 families (pre-miR156b/i, pre-miR159b/f, pre-miR166a/b/c, pre-miR167b/g, pre-miR169f/p) were up-regulated while others (pre-miR156d/f/g/j, pre-miR159a, pre-miR166d, pre-miR167d/e, pre-miR169a/b/h/l/m/q) were downregulated by drought stress. These results suggest that members of the same miRNA family are functionally diverse during drought responses. A number of droughtresponsive miRNAs have been identified [32-34]; however, we found that the expression patterns of 14 pre-miRNAs (miR156i/b/f, miR168a, miR172a/d and miR169a/b/h/l/m/ q, miR171e, miR393b) were different under the drought treatments used in this study from those previously reported $[10,15]$. This discrepancy may be due to different ages of the tissues used or the way in which the drought treatments were imposed. It is also possible that the expression patterns between the mature miRNAs and premiRNAs are different during drought conditions.

Experimental validation of the putative miRNA paired target genes has been a major focus in the investigation of miRNA function [35]. Of the differentially expressed precursor miRNAs and their putative target genes, 5 were confirmed by qRT-PCR (Fig. 2), and the expression level of these genes confirmed the accuracy of the RNAseq data. Under drought-stress conditions, the transcription of stress-responsive miRNAs and their putative targets can be independently regulated. Indeed, the expression patterns of some of the pre-miRNAs were positively correlated with those of their target genes (Additional file 8: Tabular data 2). Conversely, many drought-responsive miRNAs and their targets showed a negative correlation in their expression patterns, i.e., drought-induced miRNAs downregulated their target mRNAs, while drought-repressed miRNAs upregulated their target mRNAs (Fig. 2 and Additional file 8: Tabular data 2). Thus, a given target gene may either promote or suppress processes during stress adaptation responses. Drought-responsive miRNA-mediated target cleavages were also confirmed by analyzing publically available PARE data (Table 1). Some miRNAs, such as miR819d, miR171f, miR156, miR530 and miR819i, have a large number of putative target genes. Additional file 8: Tabular data 2 shows that the candidate target genes were up- or 
down-regulated in an opposite manner to the change in miRNA expression, e.g., miR171f and its targets, Scarecrow-like 6 and the MORN motif containing protein, and miR156 and its target squamosa-promoter binding protein. miR171f has previously been observed to be responsive to salt, drought and cold stress in A. thaliana [36], and two mature miR171fs, miR171f-3p and miR171f$5 \mathrm{p}$, were reported to be down-regulated under drought conditions in rice [33]. However, our results showed that the pre-miR171f and the mature miR171f-5p, but not miR171f-3p, were drought-inducible and that the expression level of the candidate target gene, Os03g0828701-00, was inversely correlated with that of miR171f-5p under drought conditions. The negative-correlation patterns between miR171f and Os03g0828701-00 were also found at various developmental stages of rice (Fig. 3), thereby providing insights into the function of miR171f, especially miR171f-5p, in the adaptive response of plants to drought stress at various development stages. Pre-miRNA expression profiling with RNA-seq and qRT-PCR of various developmental stages reveals that miR171f is involved in rice root growth and development as well as in responses to drought. Recently, it has been shown that virus infection specifically induces miR17f-5p expression in rice [32]. Given that mature the miR171f-3p sequence is also encoded by several other members of the miR171 family and that it is conserved to recognize target genes encoding GRAS family transcription factors, the pre-miR171f / Os03g0828701-00 module may be specifically developed to be involved in drought responses, developmental processes, and resistance to viral infection.

It was recently reported that a total of 37,238 long noncoding natural antisense transcripts (lncNATs) are associated with $70 \%$ of the annotated mRNAs in A. thaliana [37]. In addition, 125 putative stress responsive IncRNAs from wheat have been reported [24], as well as 20,163 putative maize lncRNAs [38]. Zhang et al. identified 2,224 lncRNAs by sequencing strand-specific RNAs from various rice organs, including anthers, pistils, seeds, and shoots [39]. We found a total of 98 lncRNAs whose expression changed in response to drought (31 up-regulated and 67 down-regulated) and their expression levels were positively correlated with those of their putative target genes. Interestingly, two lncRNAs, Os02g0250700-01 and Os02g0180800-01, are bidirectional, and their potential targets are present on the neighboring opposite strand. Os02g0250700-01 and its target gene, Os02g0250600-01 (late embryogenesis abundant protein; LEA) was shown to share a single bidirectional promoter, and their expression is inversely correlated, as was the expression of $\mathrm{Os} 02$ g0180800-01 and its target gene, Os02g0180700-01 (cinnamoyl-CoA reductase) under drought conditions.

Noncoding RNAs have been identified in many plant species, such as $A$. thaliana, maize, wheat, soybean
(Glycine $\max$ ) and rice; however, functional analysis is still challenging. Here, we identified drought responsive noncoding RNAs of the miRNA and lncRNA categories. The identification and expression pattern analysis of the rice precursor miRNAs and lncRNAs represents a resource for investigating how the extensive set of noncoding RNAs in the genome function and interact during drought stress and in regulating development.

\section{Conclusions}

In this current study, we identified drought responsive noncoding RNAs by using RNA-seq profiling on wellwatered control and drought-treated rice plants. A total of 66 drought-responsive miRNA precursors (24 upregulated and 42 down-regulated), which have not previously been characterized in rice, were identified. The expression levels of some of these were shown to have a negative correlation with those of their candidate target genes. Those miRNA/target pairs were further validated by Parallel Analysis of RNA Ends (PARE) libraries from various rice tissues that enabled us to identify decay intermediates generated by miRNA-guided cleavages. In addition, a total of 98 drought-responsive lncRNAs (31 drought-inducible and 67 drought-repressible) and their sense or antisense transcripts were detected. The combined data sets suggest potential roles for specific rice noncoding RNAs under drought conditions.

\section{Methods}

\section{Plant materials and drought-stress treatment}

Rice (Oryza sativa cv. Ilmi) was germinated on MS (Murashige and Skoog) media at $28{ }^{\circ} \mathrm{C}$ for 4 days, and transplanted into soil pots $(4 \times 4 \times 6 \mathrm{~cm}$; 3 plants per pot) and grown in a greenhouse $\left(37^{\circ} 32^{\prime} 51.3^{\prime \prime} \mathrm{N} 128^{\circ} 26^{\prime} 26.6^{\prime \prime} \mathrm{E}\right)$. Multiple pots of each rice were divided into 2 sets, one for drought and one for well-watered treatment conditions. Five weeks after transplanting to soil, total leaves of 10 whole plants growing in 4 pots were pooled and kept in liquid nitrogen for $\mathrm{C}$, and then water was withheld from all the pots. The soil water content in each pot was adjusted to approximately $75 \%$. Soil moisture was monitored during the drought treatment using a Soil Moisture Sensor SM150 (Delta-T Devices, UK). After one, two and three days without watering, total leaves of 10 whole plants growing in 4 pots were pooled and kept in liquid nitrogen for $1 \mathrm{~d}, 2 \mathrm{~d}$, and $3 \mathrm{~d}$, respectively. Total RNA was extracted from pooled leaves of C, $1 \mathrm{~d}, 2 \mathrm{~d}$, or $3 \mathrm{~d}$. We grew an independent group of plants similar to above, and measured water content and expression levels of drought responsive marker genes using qRT-PCR as shown in Additional file 11: Figure S4. As a result, we prepared 2 independent sets of plants for drought treatments; one for RNA-seq (Fig. 1) and the other for qRT-PCR validation (Additional file 11: Figure S4). 


\section{RNA extraction, RNA-seq library construction and sequencing}

Total RNA was extracted from rice leaves using Trizol reagent (Invitrogen) and purified with an RNeasy Mini Kit (Qiagen). Contaminating genomic DNA was removed from the sample by treating with DNase I (Invitrogen), according to the manufacturer's instructions. A modified TruSeq method was used to construct strandspecific RNA-seq libraries, with different index primers [40], and libraries were sequenced with an Illumina HiSeq 2500 system at the National Instrumentation Center of Environmental Management College of Agriculture and Life Science, Seoul National University (NICEM), as previously described [40]. Single-end sequences were generated and raw sequence reads were trimmed to remove adaptor sequences, and those with a quality lower than Q20 were removed using the clc quality trim software (CLCBIO). Duplicate paired short reads were removed using FastUniq [41], and all reads were assembled with the clc_ref_assemble 6 (version 4.06) program, using annotated gene and noncoding RNA sequences from the rapdb (http://rapdb.dna.affrc.go.jp) and ncRNA (http:// www.ncrna.org) databases, respectively. The data set can be obtained from GEO database with series accession number GSE80811 for RNA-seq data.

\section{Quantitative RT-PCR validation of transcript abundance}

One $\mu \mathrm{g}$ total RNA was reverse transcribed with oligo dT primers using $200 \mathrm{U}$ of the RevertAid M-MuLV Reverse Transcriptase (Thermo Scientific, \#K1621) for $60 \mathrm{~min}$ at $42{ }^{\circ} \mathrm{C}$, and then the reaction was terminated by incubating for $5 \mathrm{~min}$ at $70{ }^{\circ} \mathrm{C}$. Subsequent qRT-PCR was performed with first-strand $\mathrm{CDNA}$ as a template using gene-specific primer pairs and 2x Real-Time PCR smart mix (SolGent, SRH72-M10h) with EvaGreen (SolGent, 31000-B500). Reactions were performed at $95{ }^{\circ} \mathrm{C}$ for $15 \mathrm{~min}$, followed by 40 cycles of $95^{\circ} \mathrm{C}$ for $20 \mathrm{~s}, 60{ }^{\circ} \mathrm{C}$ for $20 \mathrm{~s}$, and $72{ }^{\circ} \mathrm{C}$ for $30 \mathrm{~s}$, in a $20 \mu \mathrm{l}$ reaction mixture containing $1 \mu \mathrm{l}$ of $20 \mathrm{x}$ EvaGreen, $10 \mu \mathrm{M}$ primers, and ROX reference dye. Thermocycling and fluorescence detection were performed uisng a Strategene Mx300p realtime PCR machine and Mx3000p software version 2.02 (Stratagene). The Ubi1 (AK121590) gene was used to verify equal RNA loading for the qRT-PCR analysis and as a reference in the RT-PCR. For detecting and quantifying mature miRNAs, stem-loop reverse transcription and RT-PCR of miRNAs was performed as described in Varkonyi-Gasic et al. and Chen et al. [42, 43]. Two hundred ng of total RNA was treated with RNAase-free DNase I (Promega), and transcribed into cDNA using gene specific RT primers and a thermostable reverse transcriptase (Invitrogen). First, the miRNA-specific stem-loop RT primer was hybridized to the miRNA and reverse transcribed. Reactions were performed at $16{ }^{\circ} \mathrm{C}$ for $45 \mathrm{~min}$, followed by 60 cycles of $30{ }^{\circ} \mathrm{C}$ for $45 \mathrm{~s}, 42{ }^{\circ} \mathrm{C}$ for $45 \mathrm{~s}$, and $50{ }^{\circ} \mathrm{C}$ for $1 \mathrm{~s}$, in a $20 \mu \mathrm{l}$ mixture containing 50 U Superscript III RT (Invitrogen), 4 U RNaseOUT (Invitrogen) and $1 \mu \mathrm{M}$ stem-loop RT primer. Next, RT products were quantified using qRT-PCR with a miRNA specific forward and universal reverse primer. The rice U6 small nuclear RNA (snRNA) gene was used to verify equal RNA loading for the qRT-PCR analysis and as a reference. A list of primers used in these experiments is available in Additional file 12: Table S5. All qRT-PCR results are representative from at least two biological repeats, each based on three technical repeats.

\section{Additional files}

Additional file 1: Table S1. Information about the RNA-seq data obtained by Illumina Hi-seq 2500 sequencing. (XLSX 12 kb)

Additional file 2: Figure S1. Flowchart of RNA-seq analysis. (TIF 9814 kb) Additional file 3: Figure S2. Heat Map of the differentially expressed coding (a) and noncoding genes (b) under drought conditions. (TIF 5474 kb) Additional file 4: Tabular data 1. Drought responsive genes and their expression patterns under well-watered and drought conditions. (XLSX 8528 kb)

Additional file 5: Figure S3. Differentially expressed transcripts were classified into 3 main GO categories: Biological processes, Cellular components and Molecular functions. (TIF $8355 \mathrm{~kb}$ )

Additional file 6: Table S3. List of miRNAs that are constitutive pattern of high expression and low expression level. ${ }^{1}$ Bold in miRNAs ID, rice specific drought responsive miRNAs; ${ }^{2}$ RPKM, Reads Per Kilobase of transcript per Million mapped reads; ${ }^{3} \mathrm{C}$, control; ${ }^{4} 1 \mathrm{~d}$, drought treatment for 1 day; ${ }^{5} 2 \mathrm{~d}$, drought treatment for 2 days; ${ }^{6} 3 \mathrm{~d}$, drought treatment for 3 days; ${ }^{7} \log 2$ ratio, $\log _{2}$ (drought treatment / control), ${ }^{8}$ Red, upregulation by drought; Blue, down-regulation by drought Abbreviations: At, Arabidopsis thaliana; Bd, Brachypodium distachyon; Gm, Glycine max; $\mathrm{Hv}$, Hordeum vulgare; Mt, Medicago truncatula; Me, Manihot esculenta; Pv, Phaseolus vulgaris; Peu, Populus euphratica; Ptc, Populus trichocarpa; Ppe, Prunus persica; Pte: Populus tremula; Pto, Populus tomentosa; Td, Triticum dicoccoides; Tt, Triticum turgidum; Os, Oryza sativa; Vu, Vigna unguiculata; Zm, Zea mays $[10,15]$. (XLSX $24 \mathrm{~kb})$

Additional file 7: Table S2. Drought responsive precursor miRNAs and their expression patterns under well-watered and drought conditions. Bold in miRNAs ID, rice specific drought responsive miRNAs; ${ }^{2}$ RPKM, Reads Per Kilobase of transcript per Million mapped reads; ${ }^{3} \mathrm{C}$, control (well-watered conditions); ${ }^{4} 1 \mathrm{~d}$, drought treatment for 1 day; ${ }^{5} 2 \mathrm{~d}$, drought treatment for 2 days; ${ }^{6} 3 \mathrm{~d}$, drought treatment for 3 days; ${ }^{7} \log 2$ ratio, $\log 2$ (drought treatment/control); ${ }^{8}$ Species, red letter, up-regulation by drought; blue letter, down-regulation by drought; Abbreviations: At, Arabidopsis thaliana; Bd, Brachypodium distachyon; Gm, Glycine max; Hv, Hordeum vulgare; Mt, Medicago truncatula; Me, Manihot esculenta; Os, Oryza sativa; Pv, Phaseolus vulgaris; Peu, Populus euphratica; Ptc, Populus trichocarpa; Ppe, Prunus persica; Pto, Populus tomentosa; Td, Triticum dicoccoides; Tt, Triticum turgidum; Zm, Zea mays [10, 15]. (XLSX 28 kb)

Additional file 8: Tabular data 2. List of drought responsive miRNAs and their putative target genes and their expression patterns of both under drought conditions. (XLSX $101 \mathrm{~kb}$ )

Additional file 9: Table S4. Drought responsive long noncoding RNAs with their neighboring genes and their expression patterns. (XLSX 67 kb) Additional file 10: Tabular data 3. List of drought responsive long noncoding RNAs (IncRNAs). (XLSX 529 kb)

Additional file 11: Figure S4. Drought response phenotype of rice in the vegetative state. a The phenotypic effect of progressive drought on wild type rice (Oryza sativa cv. IImi) at the vegetative growth stage. 
b Decrease in soil water content during drought treatment. c The transcript levels of Dip 1 and RbcS1 in the leaves of drought-treated and well-watered control plants over a time course of exposure to drought were measured by qRT-PCR analysis. Values shown are the means \pm SD of three independent experiments and are presented relative to the results from the control. (TIF $17024 \mathrm{~kb}$ )

Additional file 12: Table S5. Primers used for real time-PCR and the stem-loop RT-PCR miRNA assay. (XLSX $15 \mathrm{~kb}$ )

\section{Abbreviations}

IncRNAs, long noncoding RNAs; miRNAs, micro RNAs; qRT-PCR, quantitative reverse transcribed polymerase chain reaction; RNA-seq, RNA sequencing

\section{Acknowledgements}

We thanks the National Instrumentation Center of Environmental Management College of Agriculture and Life Science, Seoul National University (NICEM) for the help with the RNA-Seq and bioinformatics analyses.

\section{Funding}

This work was supported by the National Research Foundation of Korea Grant funded by the Korean Government (NRF-2013R1A6A3A04060627 to PJC). This work was also supported by the Rural Development Administration under the Next-Generation BioGreen 21 Program (Project No. PJ011829012016 to J-KK).

\section{Availability of data and materials}

The data supporting the conclusions of this article are included within the article and its additional files.

\section{Authors' contributions}

PJC and J-KK designed the research. PJC and HJ performed the molecular experiments and stress tests. PJC analyses RNA-seq. D-HJ provided and analyzed PARE data. PJC, HJ, S-HH, YDC and J-KK wrote the manuscripts. All authors read and approved the final manuscript.

\section{Competing interests}

The authors declare that they have no competing interests.

\section{Consent for publication}

Not applicable.

\section{Ethics approval and consent to participate}

Not applicable.

\section{Author details \\ ${ }^{1}$ Graduate School of International Agricultural Technology and Crop Biotechnology Institute/GreenBio Science \& Technology, Seoul National University, Pyeongchang 25354, Korea. ${ }^{2}$ Department of Agricultural Biotechnology, Seoul National University, Seoul 08826, Korea. ${ }^{3}$ Department of Life Science, Hallym University, Chuncheon 24252, Korea. ${ }^{4}$ Department of Genetic Engineering and Graduate School of Biotechnology, Kyung Hee University, Yongin 17104, Korea.}

\section{Received: 28 April 2016 Accepted: 4 August 2016}

\section{Published online: 08 August 2016}

\section{References}

1. Kang Y, Khan S, Ma X. Climate change impacts on crop yield, crop water productivity and food security. Prog Nat Sci. 2009;19(12):1665-74.

2. Lobell DB, Gourdji SM. The influence of climate change on global crop productivity. Plant Physiol. 2012;160(4):1686-97.

3. Micheletto S, Rodriguez-Uribe L, Hernandez R, Richins RD, Curry V, O'Connell MA. Comparative transcript profiling in roots of Phaseolus acutifolius and P. vulgaris under water deficit stress. Plant Sci. 2007;173(5):510-20.

4. Todaka D, Nakashima K, Shinozaki K, Yamaguchi-Shinozaki K. Toward understanding transcriptional regulatory networks in abiotic stress responses and tolerance in rice. Rice. 2012;5(1):6.

5. Lee HY, Jang G, Um T, Kim JK, Lee JS, Choi YD. The soluble ABA receptor PYL8 regulates drought resistance by controlling $A B A$ signaling in Arabidopsis. Plant Biotechnol Rep. 2015;9(5):319-30.
6. Park SH, Jeong JS, Lee KH, Kim YS, Choi YD, Kim JK. OsbZIP23 and OsbZIP45, members of the rice basic leucine zipper transcription factor family, are involved in drought tolerance. Plant Biotechnol Rep. 2015;9(2):89-96.

7. Todaka D, Shinozaki K, Yamaguchi-Shinozaki K. Recent advances in the dissection of drought-stress regulatory networks and strategies for development of drought-tolerant transgenic rice plants. Front Plant Sci. 2015;6:84.

8. Cheong YH, Chang HS, Gupta R, Wang X, Zhu T, Luan S. Transcriptional profiling revels novel interactions between wounding, pathogen, abiotic stress, and hormonal responses in Arabidopsis. Plant Physiol. 2002; 129(2):661-77.

9. Kim ED, Sung S. Long noncoding RNA: unveiling hidden layer of gene regulatory networks. Trends Plant Sci. 2012;17(1):16-21.

10. Sunkar R, Li YF, Jagadeeswaran G. Functions of microRNAs in plant stress responses. Trends Plant Sci. 2012;17(4):196-203.

11. Watanabe KA, Ringler P, Gu L, Shen QJ. RNA-sequencing reveals previously unannotated protein- and microRNA-coding genes expressed in aleurone cells of rice seeds. Genomics. 2014;103(1):122-34.

12. Yoshida T, Mogami J, Yamaguchi-Shinozaki K. ABA-dependent and ABAindependent signaling in response to osmotic stress in plants. Curr Opin Plant Biol. 2014;21:133-9.

13. Khraiwesh B, Zhu JK, Zhu J. Role of miRNAs and siRNAs in biotic and abiotic stress responses of plants. Biochim Biophys Acta. 2012;1819(2):137-48.

14. Kozomara A, Griffiths-Jones S. miRBase: annotating high confidence microRNAs using deep sequencing data. Nucleic Acids Res. 2014;42:D68-73.

15. Ferdous J, Hussain SS, Shi BJ. Role of microRNAs in plant drought tolerance. Plant Biotechnol J. 2015;13(3):293-305.

16. Zhou L, Liu Y, Liu Z, Kong Z, Duan M, Luo L. Genome-wide identification and analysis of drought-responsive microRNAs in Oryza sativa. J Exp Bot. 2010;61(15):4157-68.

17. Ding $Y$, Tao $Y$, Zhu $C$. Emerging roles of microRNAs in the mediation of drought stress response in plants. J Exp Bot. 2013;64(11):3077-86.

18. Li YF, Zheng Y, Addo-Quaye C, Zhang L, Saini A, Jagadeeswaran G, Axtell MJ, Zhang W, Sunkar R. Transcriptome-wide identification of mircoRNA targets in rice. Plant J. 2010;62(5):742-59.

19. Li WX, Oono Y, Zhu J, He XJ, Wu JM, lida K, Lu XY, Cui X, Jin H, Zhu JK. The Arabidopsis NFYA5 transcription factor is regulated transcriptionally and posttranscriptionally to promote drought resistance. Plant Cell. 2008:20(8):2238-51.

20. Jeong DH, Green PJ. The role of rice microRNAs in abiotic stress responses. J Plant Biol. 2013;56(4):187-97.

21. Jeong DH, Park S, Zhai J, Gurazada SG, De Paoli E, Meyers BC, Green PJ. Massive analysis of rice small RNAs: mechanistic implication of regulated microRNAs and variants for differential target RNA cleavage. Plant Cell. 2011 23(12):4185-207.

22. Ponting CP, Oliver PL, Reik W. Evolution and functions of long nonoding RNAs. Cell. 2009;136(4):629-41.

23. Liu J, Jung C, Xu J, Wang H, Deng S, Bernad L, Arenas-Huertero C, Chua NH. Genome-wide analysis uncovers regulation of long intergenic noncoding RNAs in Arabidopsis. Plant Cell. 2012;24(11):4333-45.

24. Xin M, Wang $Y$, Yao $Y$, Song $N$, Hu Z, Qin D, Xie C, Peng H, Ni Z, Sun Q. Identification and characterization of wheat long non-protein coding RNAs responsive to powdery mildew infection and heat stress by using microarray analysis and SBS sequencing. BMC Plant Biol. 2011;11:61.

25. Zhang W, Han Z, Guo Q, Lin Y, Zheng Y, Wu F, Jin W. Identification of maize long non-coding RNAs responsive to drought stress. PLoS One. 2014;9(6):e98958.

26. German MA, Luo S, Schroth G, Meyers BC, Green PJ. Construction of parallel analysis of RNA ends (PARE) libraries for the study of cleaved miRNA targets and the RNA degradome. Nature Protoc. 2009;4(3):356-62.

27. Park SH, Chung PJ, Juntawong P, Bailey-Serres J, Kim YS, Jung H, Bang SW, Kim YK, Choi YD, Kim JK. Posttranscriptional control of photosynthetic mRNA decay under stress conditions requires $3^{\prime}$ and $5^{\prime}$ untranslated regions and correlates with differential polysome association in rice. Plant Physiol. 2012;159(3):1111-24.

28. Chung PJ, Kim YS, Park SH, Nahm BH, Kim JK. Subcellular localization of rice histone deacetylases in organelles. FEBS Lett. 2009;583(13):2249-54.

29. Hou CY, Wu MT, Lu SH, Hsing YI, Chen HM. Beyond cleaved small RNA targets: unraveling the complexity of plant RNA degradome data. BMC Genomics. 2014:15:15

30. Cai S, Jiang G, Ye N, Chu Z, Xu X, Zhang J, Zhu G. A key ABA catabolic gene, OsABA80x3, is involved in drought stress resistance in rice. PLoS One. 2015;10(2):e0116646. 
31. Campo S, Baldrich P, Messeguer J, Lalanne E, Coca M, San Segundo B. Overexpression of a calcium-dependent protein kinase confers salt and drought tolerance in rice by preventing membrane lipid peroxidation. Plant Physiol. 2014;165(2):688-704.

32. Du P, Wu J, Zhang J, Zhao S, Zheng H, Gao G, Wei L, Li Y. Viral infection induces expression of novel phased microRNAs from conserved cellular microRNA precursors. PLoS Pathog. 2011;7(8):e1002176.

33. Qin J, Ma X, Tang Z, Meng Y. Construction of regulatory networks mediated by small RNAs responsive to abiotic stresses in rice (Oryza sativa). Comput Biol Chem. 2015;58:69-80.

34. Zhao B, Liang R, Ge L, Li W, Xiao H, Lin H, Ruan K, Jin Y. Identification of drought-induced microRNAs in rice. Biochem Biophys Res Commun. 2007; 354(2):585-90

35. Thomson DW, Bracken CP, Goodall GJ. Experimental strategies for microRNA target identification. Nucleic Acids Res. 2011;39(16):6845-53.

36. Liu HH, Tian X, Li YJ, Wu CA, Zheng CC. Microarray-based analysis of stressregulated microRNAs in Arabidopsis thaliana. RNA. 2008;14(5):836-43.

37. Wang H, Chung PJ, Liu J, Jang IC, Kean MJ, Xu J, Chua NH. Genome-wide identification of long noncoding natural antisense transcripts and their responses light in Arabidopsis. Genome Res. 2014;24(3):444-53.

38. Li L, Eichten SR, Shimizu R, Petsch K, Yeh CT, Wu W, Chettoor AM, Givan SA Cole RA, Fowler JE, Evans MM, Scanlon MJ, Yu J, Schnable PS, Timmermans MC, Springer NM, Muehlbauer GJ. Genome-wide discovery and characterization of maize long noncoding RNAs. Genome Biol. 2014;15(2):R40.

39. Zhang YC, Liao JY, Li ZY, Yu Y, Zhang JP, Li QF, Qu LH, Shu WS, Chen YQ. Genome-wide screening and functional analysis identify a large number of long noncoding RNAs involved in the sexual reproduction of rice. Genome Biol. 2014;15(12):512

40. Zhong S, Joung JG, Zheng Y, Chen YR, Liu B, Shao Y, Xiang JZ, Fei Z, Giovannoni JJ. High-throughput illumine strand-specific RNA sequencing Library preparation. Cold Spring Harb Protoc. 2011;8:940-9.

41. Xu H, Luo X, Qian J, Pang X, Song J, Qian G, Chen J, Chen S. FastUniq: A fast de novo duplicates removal tool for paired short reads. PLoS One. 2012; 7(12):e52249.

42. Varkonyi-Gasic E, Wu R, Wood M, Walton EF, Hellens RP. Protocol: a highly sensitive RT-PCR method for detection and quantification of microRNAs. Plant Methods. 2007;3:12

43. Chen C, Ridzon DA, Broomer AJ, Zhou Z, Lee DH, Nguyen JT, Barbisin M, Xu NL, Mahuvakar VR, Adersen MR, Lao KQ, Livak KJ, Guegler KJ. Real-time quantification of microRNAs by stem-loop RT-PCR. Nucleic Acids Res. 2005;33(20):e179.

\section{Submit your next manuscript to BioMed Central and we will help you at every step:}

- We accept pre-submission inquiries

- Our selector tool helps you to find the most relevant journal

- We provide round the clock customer support

- Convenient online submission

- Thorough peer review

- Inclusion in PubMed and all major indexing services

- Maximum visibility for your research

Submit your manuscript at www.biomedcentral.com/submit 